\title{
Prostate/Seminal Vesicles
}

National Cancer Institute

\section{Source}

National Cancer Institute. Prostate/Seminal Vesicles. NCI Thesaurus. Code C77670.

A laboratory specimen consisting of the prostate and seminal vesicles. 